University of Zurich

Department of Economics

Working Paper Series

ISSN 1664-7041 (print)

ISSN 1664-705X (online)

Working Paper No. 302

\title{
Strictly Sincere Best Responses under Approval Voting and Arbitrary Preferences
}

Carlos Alós-Ferrer and Johannes Buckenmaier

October 2018 


\title{
Strictly Sincere Best Responses under Approval Voting and Arbitrary Preferences*
}

\author{
Carlos Alós-Ferrer ${ }^{\dagger}$ \\ Johannes Buckenmaier \\ University of Zurich \\ University of Zurich
}

This Version: August, 2018

\begin{abstract}
Approval voting allows voters to support as many candidates as they wish. One advantage of the method is that voters have weak or no incentives to vote insincerely. However, the exact meaning of this statement depends on how the voters' preferences over candidates are extended to sets. We show that, under a combination of standard, well-established assumptions on the extended preferences, voters will always have a strictly sincere best response (that is, a best response ballot such that every approved candidate is strictly preferred to every disapproved one) given the ballots of other voters. The result holds for arbitrary preferences over candidates, allowing for indifferences but covering the extreme cases of dichotomous or strict preferences. As a corollary, we show that the classical strategy-proofness result for the case of dichotomous preferences on alternatives (Brams and Fishburn, 1978) holds for a larger class of preferences on sets than originally assumed.
\end{abstract}

JEL Classification: C72 - D71 - D72

Keywords: Approval voting - Manipulation - Preferences among sets . Strict sincerity

Working Paper. This is an author-generated version of a research manuscript which is circulated exclusively for the purpose of facilitating scientific discussion. All rights reserved. The final version of the article might differ from this one.

*Financial support from the German Research Foundation (DFG) through project AL-1169/2 is gratefully acknowledged.

${ }^{\dagger}$ Corresponding author: carlos.alos-ferrer@econ.uzh.ch. Department of Economics, University of Zurich. Blümlisalpstrasse 10, 8006 Zurich, Switzerland.

${ }^{\ddagger}$ Department of Economics, University of Zurich. Blümlisalpstrasse 10, 8006 Zurich, Switzerland. 


\section{Introduction}

Approval voting is a voting method where voters are allowed to approve of as many candidates as they wish. Since its introduction by Brams and Fishburn (1978), a number of appealing properties of this method have been discussed. Among the intuitive properties are that the support for vocal extremists is greatly reduced, as shown e.g. in the field experiments of Laslier and Van der Straeten (2008) and AlósFerrer and Granić (2012, 2015) (see also Baujard and Igersheim, 2010; Alós-Ferrer and Granić, 2010). Also, the method is less susceptible to polarize the electorate and lead candidates to run "smear campaigns," simply because candidates might partly share their voter base. Among the more formal properties, Approval Voting has been characterized as the only method aggregating multi-candidate ballots which fulfills a number of appealing properties (Fishburn, 1978a,b; Alós-Ferrer, 2006). Last but most definitely not least, it has been argued that the Approval Voting method is less vulnerable (or not at all) to voter manipulation (Brams and Fishburn, 1978, 2005; Wolitzky, 2009). ${ }^{1}$

The intuition for this latter property is simple. Most reasons for strategic voter behavior adopt a similar form. The voter would like to support candidate $a$, but given the belief on the decisions of other voters, it is unlikely that candidate $a$ will win, and supporting that candidate comes at the cost of not being able to support candidate $b$, which is not as good for the voter as $a$, but definitely better than candidate $c$, who has clear chances of being elected. Hence, a vote for $a$ becomes a "wasted vote" and the voter is forced to misrepresent his or her preferences. The wasted-vote effect creates a catch-22 where small parties remain small because they are seen as small, and big traditional parties receive a large support simply because they are seen as big. This effect might be easily seen to contribute to creating a disconnect between the electorate's true preferences and the elected parties' positions.

Approval Voting escapes the "wasted vote" effect. Even if the voter decides to support $b$ in order to avoid $c$, there is no reason not to support $a$, because this latter decision comes at no cost. The voter can simply cast the ballot $\{a, b\}$, which is sincere. The "strategic" motive has simply caused the voter to select one among several possible sincere votes, where sincerity is defined as preferring each approved candidate to each disapproved one. A formalization of the idea, how-

\footnotetext{
${ }^{1}$ Because the voter's non-strategic decision cannot be uniquely derived from preferences alone, Approval Voting does not belong to the family of voting methods covered by the GibbardSatterthwaite Theorem, which states that all methods (in that family) are manipulable (Gibbard, 1973; Satterthwaite, 1975).
} 
ever, is not straightforward. The reason is that sincerity is a property of ballots, which under Approval Voting are sets of candidates, and whether a sincere ballot is selected or not is an assertion on whether it is preferred to other ballots. The standard formal framework in social choice starts with preferences over candidates (or alternatives). Hence, to evaluate whether one ballot is preferred to another, one needs to extend those preferences to sets of candidates. However, given a fixed preference relation among candidates, there are many different extensions to sets of candidates. Depending on which properties are deemed reasonable for such an extension, sincere ballots might be preferred to insincere ones or not. The literature has examined many different properties of extended preferences, always with the aim of identifying the extent of non-manipulability of Approval Voting. For instance, Brams and Fishburn (1978) showed that Approval Voting is nonmanipulable under two conditions on the extended preferences (conditions $(\mathrm{R}$ ) and $(\mathrm{P})$ ), but their result was restricted to the class of dichotomous preferences. Recently, Endriss (2013) identified a collection of conditions guaranteeing that a voter will always have a sincere best response, given the ballots of other voters, but the result was restricted to strict preferences over candidates (no indifferences allowed). However, De Sinopoli et al. (2006) showed an example of a Nash equilibrium in mixed strategies where one player has a unique best response which is insincere, for a specific preference extension based on risk-neutral evaluation of random tie-breaking if the set of winners contains more than one candidate.

In this work, we provide a simple formalization of the idea that Approval Voting is resistant to strategic misrepresentation of preferences. We explicitly allow for general preferences, including dichotomous ones as in Brams and Fishburn (1978), strict preferences as in Endriss (2013), and the general case of non-strict preferences.

Our result is based on three conditions, all of which are already present in the literature. The first is condition (R) from Brams and Fishburn (1978), which, in our view is a monotonicity property. We show that this condition is equivalent to the following two properties: i) adding new options to a ballot which are better than all alternatives already in the ballot produces at least a weak improvement; ii) dropping some options from the ballot which were weakly worse than every other alternative remaining in the ballot produces at least a weak improvement.

The other two properties were introduced by Endriss (2013) for the case of strict preferences, and we consider their extensions to the general case. A preference extension fulfills deletion if removing a candidate from a set yields a weak improvement whenever this set is strictly preferred to obtaining only that candidate 
alone. Further, a preference extension fulfills replacement if whenever some alternative is preferred to an alternative already in the set, then a weak improvement over the latter set can be obtained by either exchanging those two elements, adding the former to the set, or removing the latter from the set. Under monotonicity, deletion, and replacement, we prove that every voter has a sincere best response given the ballots of other voters. Even more, our result is actually stronger because what is proven is the existence of a strictly sincere best response, that is, one where each approved candidate is strictly preferred to each disapproved candidate.

Interestingly, in the case of dichotomous preferences studied by Brams and Fishburn (1978), condition (R) implies both deletion and replacement. It then follows immediately from our result that, in this case, sincere voting is strategy proof under Approval Voting for any preference extension to sets satisfying condition (R). Brams and Fishburn (1978) proved this result under two conditions, (R) and $(\mathrm{P})$. The latter is somewhat restrictive, being inspired by extensions where sets of winners are evaluated in a risk-neutral way as if a final winner would be selected at random. Our analysis implies that condition $(\mathrm{P})$ is unnecessary for the strategy-proofness result, an observation of obvious independent interest.

The article is structured as follows. Section 2 introduces the formal framework and the concepts of sincere and strictly sincere ballot. Section 3 introduces the properties we focus on and related ones. Section 4 proves the main result. Section 5 reexamines the case of dichotomous preferences and shows that the classical result of Brams and Fishburn (1978) holds with just one of their two assumptions. Section 6 establishes the relations between our conditions and the ones previously studied in the literature. Section 7 concludes.

\section{Formal framework}

The notation in this section essentially follows Alós-Ferrer (2006). Let $X$ be a finite set of at least two alternatives. A ballot is a subset of alternatives, $B \subseteq X$. Let $\mathcal{B}=\mathcal{P}(X)$ be the set of admissible ballots. Voters can cast any ballot, approving of as many candidates as they want, but cannot reveal any preference among the candidates they approve (or disapprove) of. The empty and the full ballot are allowed, that is, voters can abstain. A ballot $B$ is nontrivial if $\emptyset \neq B \neq X$.

A voter response profile is a function $\pi: \mathcal{B} \mapsto \mathbb{N}$, with the interpretation that $\pi(B)$ is the number of voters who cast ballot $B$. The number of participating voters is $\sum_{B \in \mathcal{B}} \pi(B)$. Let $\Pi$ be the set of all possible voter response profiles (implicitly, this allows for variable electorate size), including the empty profile $\pi^{\emptyset}$ 
such that $\pi^{\emptyset}(B)=0$ for all $B \in \mathcal{B}$. Given $x \in X$ and $\pi \in \Pi$, denote $v(x, \pi)=$ $\sum\{\pi(B) \mid x \in B \in \mathcal{B}\}$, i.e. the number of voters who approve of $x$ in $\pi$. Approval Voting is a ballot aggregation function, that is, a correspondence $f$ which assigns to every possible voter response profile $\pi \in \Pi$ a nonempty set of selected alternatives, $\emptyset \varsubsetneqq f(\pi) \subseteq X$. Specifically, it is the function which selects the maximizers of $v(\cdot, \pi)$ as winners,

$$
f^{A}(\pi)=\arg \max _{x \in X} \sum\{\pi(B) \mid x \in B \in \mathcal{B}\} .
$$

Let $\mathcal{A}$ be the set of all possible preferences on $X$. Given a preference $\succeq \in \mathcal{A}$, and following Brams and Fishburn (1978), a ballot $B \in \mathcal{B}$ is called sincere if, whenever $x \in B$ and $y \succ x$, it follows that $y \in B$. We will deal with a more stringent concept (and hence obtain a stronger result). We call a ballot $B \in \mathcal{B}$ strictly sincere if $x \succ y$ for all $x \in B$ and all $y \in X \backslash B$, where $\succ$ is the strict preference associated to $\succeq$. Equivalently, $B$ is strictly sincere if, whenever $x \in B$ and $y \succeq x$, it follows that $y \in B$.

Two comments are in order. First, the empty and the full ballots are trivially strictly sincere, but, since those capture abstention, it is interesting to ask when nontrivial (strictly) sincere best responses exist, and hence there are incentives for voter participation. For this purpose, we will distinguish between trivial and nontrivial best responses. Second, if the preferences of a particular voter are strict, that is, the voter is never indifferent among two different candidates, every sincere ballot is strictly sincere (and, obviously, vice versa). For general preferences, however, strict sincerity is a more stringent concept than sincerity.

Given a voter with preferences over candidates given by $\succeq \in \mathcal{A}$ and a ballot $B \in \mathcal{B}$, let the upper set of $B$ be given by

$$
U_{\succeq}(B)=\{x \in X \mid x \succeq b \forall b \in B\}
$$

that is, the set of all candidates that are viewed as (weakly) better than any and all candidates in the ballot $B$. For notational convenience, let the indifference class of an element $a \in X$ be denoted by

$$
I_{\succeq}(a)=\{x \in X \mid x \sim a\}
$$

Note that $B \cap U_{\succeq}(B)$ is the set of maxima of $\succeq$ restricted to $B$, denoted by $\max _{\succeq}(B)$ or simply (if no confusion can arise) by $\max B$. Also, for any ballot $B$ and any preference $\succeq$, the set $U_{\succeq}(B)$ is always strictly sincere. For, if $x \in U_{\succeq}(B)$ and $y \notin U_{\succeq}(B)$, then if $B \neq \emptyset$ there exists $z \in B$ such that $x \succeq z \succ y$, and hence $x \succ y$. If $B=\emptyset$, then $U_{\succeq}(B)=X$, which is strictly sincere. 
Voters are endowed with individual preferences over alternatives $\succeq_{i} \in \mathcal{A}$. Since Approval Voting delivers sets of winners, however, we need to consider preferences over non-empty subsets of $X$, i.e. $\mathcal{P}(X) \backslash\{\emptyset\}$. To avoid unnecessarily complex notation, we will use the same symbols $(\succeq, \succ)$ for the preference over sets and the preference over alternatives. Since they apply to different formal objects, no confusion should arise.

\section{Conditions on preferences over sets}

Consider a voter with preferences over candidates given by $\succeq \in \mathcal{A}$. In their seminal article, Brams and Fishburn (1978, p.833) consider preference extensions based on two assumptions.

(R) For any $X_{1}, X_{2}, X_{3} \in \mathcal{B}$, if $X_{1} \cup X_{2} \neq \emptyset \neq X_{2} \cup X_{3}$ and $x_{1} \succeq_{i} x_{2}, x_{2} \succeq_{i} x_{3}$, and $x_{1} \succeq_{i} x_{3}$ for all $x_{1} \in X_{1}, x_{2} \in X_{2}$, and $x_{3} \in X_{3}$, then $X_{1} \cup X_{2} \succeq X_{2} \cup X_{3}$.

(P) For any $x, y \in X$, if $x \succ_{i} y$, then $\{x\} \succ\{x, y\} \succ\{y\}$.

We first disentangle condition $(\mathrm{R})$ in two separate properties for analytical convenience. We say that a preference on sets of candidates $\succeq$ fulfills monotonicity if the following two conditions hold.

(MN1) For all ballots $A, B$ such that $B \neq \emptyset$ and $A \subseteq U_{\succeq}(B)$, it follows that $A \cup B \succeq B$.

(MN2) For all ballots $A, B$ such that $B \neq \emptyset, A \subseteq B$ and $\emptyset \neq B \backslash A \subseteq U_{\succeq}(A)$, it follows that $B \backslash A \succeq B$.

Condition (MN1) states that adding new options to a ballot $B$ which are better than all alternatives previously in the ballot produces at least a weak improvement. That is, adding new candidates to a set always produces a weakly better set provided all added candidates are weakly preferred to all previous ones. Condition (MN2) states that dropping some options from the ballot $B$ which were weakly worse than every other alternative remaining in the ballot produces at least a weak improvement. That is, dropping candidates from a set always produces a weakly better set provided all dropped candidates are weakly worse than all non-dropped ones.

It turns out that monotonicity is fully equivalent to condition $(\mathrm{R})$. In the sequel, we will refer to it by the latter name since it is well-known in the literature, but rely on properties (MN1) and (MN2). 
Lemma 1. A preference relation on $\mathcal{B}$ fulfills monotonicity if and only if it fulfills condition $(R)$.

Proof. To see that monotonicity implies (R), let $X_{1}, X_{2}, X_{3}$ be as assumed in (R). First, suppose $X_{2}=\emptyset$. Thus, $X_{1}, X_{3} \neq \emptyset$. By hypothesis $X_{1} \subseteq U_{\succeq}\left(X_{3}\right)$, hence applying (MN1) to $X_{1}$ and $X_{3}$ yields $X_{1} \cup X_{3} \succeq X_{3}$ and applying (MN2) to $X_{3}$ and $X_{1} \cup X_{3}$ yields $X_{1}=\left(X_{1} \cup X_{3}\right) \backslash X_{3} \succeq X_{1} \cup X_{3}$. By transitivity it follows $X_{1} \succeq X_{3}$. Next, suppose $X_{2} \neq \emptyset$. By hypothesis $X_{2} \subseteq U_{\succeq}\left(X_{3}\right)$ and applying (MN2) to $X_{3}$ and $X_{2} \cup X_{3}$ yields $X_{2} \succeq X_{2} \cup X_{3}$. Further, $X_{1} \subseteq U_{\succeq}\left(X_{2}\right)$ by hypothesis and applying (MN1) to $X_{1}$ and $X_{2}$ we obtain $X_{1} \cup X_{2} \succeq X_{2}$. By transitivity it follows $X_{1} \cup X_{2} \succeq X_{2} \cup X_{3}$. Hence (R) holds.

To see that $(\mathrm{R})$ implies monotonicity, let $A, B \in \mathcal{B}$ with $B \neq \emptyset$. If $A \subseteq U_{\succeq}(B)$, applying (R) to $X_{1}=A, X_{2}=B$, and $X_{3}=\emptyset$ yields $A \cup B \succeq B$, proving (MN1). If $A \subseteq B$ and $\emptyset \neq B \backslash A \subseteq U_{\succeq}(A)$, applying (R) to $X_{1}=\emptyset, X_{2}=B \backslash A$, and $X_{3}=A \cap B$ yields $B \backslash A \succeq B$, proving (MN2).

The next property is the deletion axiom by Endriss (2013), which states that if a set is strictly preferred to some singleton set, then this element can be safely deleted from the set without reducing its attractiveness. In other words, if an element of a set is strictly worse than the set itself, then dropping that element from the set produces at least a weak improvement.

(DEL) For all nonempty ballots $A$ and all $a \in A$ such that $A \succ\{a\}$, it follows that $A \backslash\{a\} \succeq A$.

Finally, we will require the following replacement axiom, which is a slightly stronger version of the weak replacement axiom introduced by Endriss (2013). ${ }^{2}$ The strengthening is needed to deal with the case of indifferences and was hence not required in Endriss (2013) (which considered strict preferences only).

(RP) For every non-empty ballot $A$ and for all $a \in A$, one of the following holds.

(RP.1) $A \backslash\{a\} \succeq A$ and $A \neq\{a\}$.

(RP.2) $A \cup B \succeq A$ for each $b \in X$ with $b \succeq a$ and $B \subseteq I_{\succeq}(b)$.

(RP.3) $(A \backslash\{a\}) \cup B \succeq A$ for each $b \in X$ with $b \succ a$ and $B \subseteq I_{\succeq}(b)$, and also $A \cup B \succeq A$ for each $B \subseteq I_{\succeq}(a)$.

\footnotetext{
${ }^{2}$ This axiom in Endriss (2013) is itself a weaker version of the replacement axiom by Puppe (1995) stating that $(A \backslash\{a\}) \cup\{b\} \succeq A$ if $b \succ a$ for $a \in A$ and $b \notin A$.
} 
Essentially, condition (RP) requires that if some element $b$ is preferred to an element $a \in A$, then the set $A$ can be weakly improved by either exchanging $a$ by (a subset of the indifference class of) $b$, adding (a subset of the indifference class of) $b$ to the set, or removing $a$ from the set. Further, in case $b$ is indifferent to $a$ it requires that already one of the latter two actions results in a weak improvement. The condition reduces exactly to the replacement axiom of Endriss (2013) if one restricts the analysis to the domain of strict preferences, but allows to deal with the general case with a minimum amount of added complexity.

To put our conditions in context, we also consider a stronger but natural one. We say that a preference on sets of candidates $\succeq$ fulfills subset improvement, (SIM), if the following holds.

(SIM) For all nonempty ballots $A, B$ such that $A \backslash B \subseteq U_{\succeq}(B)$ and $A \cap U_{\succeq}(B) \neq \emptyset$, it follows that $A \succeq B$.

In words, (SIM) states that if a set of candidates is added to a subset of a ballot $B$, all of which are weakly preferred to all candidates in the full ballot, then the new ballot $A$ is necessarily seen as at least weakly better than the previous one, $B$. To put it differently, if a set $A$ consists of alternatives that were already in $B$ and at least some alternative which is better than all alternatives in $B$, then $A$ must be seen as better than $B .^{3}$

The next result shows that subset improvement implies all previously-stated conditions except for condition $(\mathrm{P})$.

Lemma 2. Given a preference on candidates, if a preference on sets of candidates fulfills (SIM), then it also fulfills condition $(R)$, deletion, and replacement.

Proof. Let $B$ be a nonempty ballot.

(MN1). Let $A \subseteq U_{\succeq}(B)$. If $A=\emptyset$, then $A \cup B \succeq B$ holds trivially. If $A \neq \emptyset$, let $C=A \cup B$. Then $C \backslash B=A \subseteq U_{\succeq}(B)$ and $C \cap U_{\succeq}(B) \supseteq A \neq \emptyset$. Applying (SIM) to $C$ and $B$, it follows that $A \cup B=C \succeq B$.

(MN2). Let $A \subseteq B$ be such that $\emptyset \neq B \backslash A \subseteq U_{\succeq}(A)$. Let $C=B \backslash A \neq \emptyset$. Then $C \backslash B=\emptyset \subseteq U_{\succeq}(B)$. By hypothesis, for every $z \in C=B \backslash A$ it follows that $z \succeq a$ for all $a \in A$. Hence $C \cap U_{\succeq}(B) \neq \emptyset$, else we would have $a \succ z$ for all $z \in B \backslash A$ and some $a \in A$, a contradiction. Applying (SIM) to $C$ and $B$, it follows that $B \backslash A=C \succeq B$.

(DEL). Let $b \in B$ such that $B \succ\{b\}$. Set $A=B \backslash\{b\}$. If $A \cap U_{\succeq}(B) \neq \emptyset$, then applying (SIM) to $A$ and $B$, it follows $A=B \backslash\{b\} \succeq B$. If $A \cap U_{\succeq}(B)=\emptyset$, then

\footnotetext{
${ }^{3} \mathrm{~A}$ possible criticism of (SIM) is that the voter then must be indifferent among all sets of mutually indifferent alternatives. This criticism is void if preferences are strict.
} 
$\max (B)=\{b\}$ and applying (SIM) to $\{b\}$ and $B$ yields $\{b\} \succeq B$, a contradiction. Hence, (DEL) holds.

(RP). Let $A \neq \emptyset$ and $a \in A$. If $a \notin U_{\succeq}(A)$, then $(A \backslash\{a\}) \cap U_{\succeq}(A) \neq \emptyset$ and $(A \backslash\{a\}) \backslash A=\emptyset \subseteq U_{\succeq}(A)$. It follows by (SIM) that $A \backslash\{a\} \succeq A$, showing that (RP.1) holds. If $a \in U_{\succeq}(A)$, let $b \in X$ with $b \succeq a$ and $B \subseteq I_{\succeq}(b)$. Then $(B \cup A) \backslash A=B \subseteq U_{\succeq}(A)$ and $(A \cup B) \cap U_{\succeq}(A) \neq \emptyset$ since $a \in(A \cup B) \cap U_{\succeq}(A)$. Applying (SIM) to $A \cup B$ and $A$ yields $A \cup B \subseteq A$, hence (RP.2) holds. Hence we have either (RP.1) or (RP.2) and (RP) holds.

Condition (SIM) is stronger than (R) because it requires $A \succeq B$ to hold even if the new candidates (preferred to every single member of $B$ ) are added to a strict subset of $B$, where some candidates have been dropped. Property (SIM), however, does not imply $(\mathrm{P})$, because the latter states strict indifference. Conceptually, however, (SIM) represents a (slightly) more restrictive condition than the conjunction of (P) and (R) used in Brams and Fishburn (1978).

Given a preference over candidates, a preference over sets of candidates is called an extension, respectively a weak extension of the former if property (EXT) below, respectively (WXT), holds.

(EXT) For all $a, b \in X$ such that $a \succ b$, it follows $\{a\} \succ\{b\}$.

(WXT) For all $a, b \in X$ such that $a \succeq b$, it follows $\{a\} \succeq\{b\}$.

Monotonicity and subset improvement are just consistency conditions between a given preference over candidates and a given preference over sets. A priori, they do not require the latter to be an extension of the former. However, it turns out that both of them actually imply (WXT).

Lemma 3. Given a preference on candidates, if a preference on sets of candidates fulfills condition (R), then it also fulfills (WXT). Hence, (SIM) also implies $(W X T)$.

Proof. Let $a, b \in X$ such that $a \succeq b$. If $a=b$, there is nothing to prove. Hence, suppose $a \neq b$. First we apply (MN1) to the nonempty ballots $\{a\}$ and $\{b\}$. Since $\{a\} \subseteq U_{\succeq}(\{b\}),(\mathrm{MN} 1)$ implies $\{a, b\} \succeq\{b\}$. Second we apply (MN2) to the nonempty ballots $\{b\}$ and $\{a, b\}$. Since $\{b\} \subseteq\{a, b\}$ and $\{a, b\} \backslash\{b\}=\{a\} \subseteq$ $U_{\succeq}(\{b\})$, (MN2) implies that $\{a\}=\{a, b\} \backslash\{b\} \succeq\{a, b\}$. Putting both properties together, we obtain $\{a\} \succeq\{a, b\} \succeq\{b\}$ and the conclusion follows from transitivity. That (SIM) also implies (WXT) follows from Lemma 2. 
In contrast, it is easy to see that neither condition $(\mathrm{R})$ together with deletion and replacement nor subset improvement imply (EXT), since the latter is stated in terms of strict preference. In order to work with extensions as captured by this more stringent condition, (EXT) needs to be assumed separately. However, this condition is not required for our main result.

Section 6 below discusses the relation of condition (R), replacement, and subset improvement to conditions previously discussed in the literature.

\section{Existence of strictly sincere best responses}

Let $I$ be a finite set of voters, each of them endowed with preferences over candidates $\succeq_{i}$. We do not assume those preferences to be strict, that is, voters might be indifferent among some candidates. We denote the ballot cast by voter $i$ by $B_{i}$. Following the standard game-theoretic convention, denote by $B_{-i}$ the vector of ballots cast by voters other than $i$. Then, $f^{A}\left(B_{i}, B_{-i}\right)$ represents the corresponding outcome of Approval Voting.

Theorem 1. Let voter $i$ have arbitrary preferences over candidates and preferences over sets fulfilling condition $(R)$, deletion, and replacement. Let $B_{-i}$ be an arbitrary vector of ballots for the other voters.

(a) There exists a strictly sincere ballot for $i$ which is a best response to $B_{-i}$.

(b) If voter $i$ 's preferences over candidates are nontrivial, then there exists a nontrivial, strictly sincere ballot for $i$ which is a best response to $B_{-i}$.

Proof. We require some preliminary notation and concepts. Let $B$ be an arbitrary ballot for $i$ and $B_{-i}$ the vector of ballots cast by voters other than $i$. Let $K$ be the highest number of votes received by a candidate under $B_{-i}$ (excluding the approvals of $i$ ). The set of pivotal candidates, denoted by $P$, is the set of candidates that received exactly $K$ votes under $B_{-i}$. The set of subpivotal candidates, denoted by $S$, is the set of candidates that received exactly $K-1$ votes under $B_{-i}$.

Note that for every $C \in \mathcal{B}$ under $\left(C, B_{-i}\right)$ an alternative can receive at most $K+1$ approval votes and alternatives in $P$ receive at least $K$ approval votes. Hence, for any $C \in \mathcal{B}$ the set of winners $W^{C}=f^{A}\left(C, B_{-i}\right)$ is a subset of $P \cup S$. More precisely, $W^{C}$ is either a subset of pivotal candidates or it is the union of all pivotal candidates and a subset of subpivotal candidates.

For $B \in \mathcal{B}$ we denote the set of approved candidates that are weakly dominated by some non-approved candidate by $\iota(B)=\left\{b \in B \mid \exists c \in X \backslash B\right.$ s.t. $\left.c \succeq_{i} b\right\}$. We 
will make use of the following relation among ballots, which allows us to order ballots by their "degree of sincerity" (Endriss, 2013). Specifically, given $B, B^{\prime} \in \mathcal{B}$, we say that $B$ is strictly more sincere than $B^{\prime}$ if and only if either $B$ is strictly sincere and $B^{\prime}$ is not, or both are not strictly sincere and one of the following conditions holds:

(S1) $b \succ b^{\prime}$ for all $b \in \max \left(X \backslash B^{\prime}\right), b^{\prime} \in \max (X \backslash B)$

(S2) $b \sim b^{\prime}$ for all $b \in \max \left(X \backslash B^{\prime}\right), b^{\prime} \in \max (X \backslash B)$ and $|\iota(B)|<\left|\iota\left(B^{\prime}\right)\right|$

The interpretation is as follows: A ballot becomes strictly more sincere if the top non-approved alternatives become less preferred ones, or if the number of approved alternatives that are dominated by some non-approved alternative becomes smaller while the top non-approved alternatives remain equally preferred.

We first establish the following useful fact.

Claim. If $b \in \iota(B)$, then $B \backslash\{b\}$ is strictly more sincere than $B$.

To see the claim, just note that $X \backslash(B \backslash\{b\})=X \backslash B \cup\{b\}$. The indifference condition in (S2) follows from this equality and the fact that $b \in \iota(B)$. The remainder of (S2) follows because $\iota(B \backslash\{b\}) \subseteq \iota(B)$ and $b \notin \iota(B \backslash\{b\})$.

We now turn to the proof of part (a). Let $B$ be a ballot for $i$ that is not strictly sincere, and let $W=f^{A}\left(B, B_{-i}\right)$ be the set of winners under Approval Voting if ballots $B$ and $B_{-i}$ are cast. We have to show that there always exists a strictly sincere ballot such that $i$ weakly prefers the outcome under that ballot to $W$. As a first step, we show that such a ballot always exists if there is a nonempty subset of pivotal candidates that is weakly preferred to $W$. Second, when no such set exists we describe an iterative procedure that allows us to construct a strictly sincere ballot that is (weakly) preferred. Thus, we distinguish the following two cases.

Case 1 . There exists a nonempty subset of pivotal candidates $P^{\prime} \subseteq P$ such that $P^{\prime} \succeq W$.

Assume such a set $P^{\prime}$ exists. Note that it follows from $(\mathrm{R})$ that $\max (P) \succeq$ $\max \left(P^{\prime}\right) \succeq P^{\prime}$ as $P^{\prime} \subseteq P$. Thus $\max (P) \succeq P^{\prime} \succeq W$, that is, $i$ weakly prefers $\max (P)$ to $W$. Casting the strictly sincere ballot $B^{*}=U_{\succeq}(\max (P))$ yields the outcome $W^{B^{*}}=\max (P)$, which is a weak improvement for $i$. Hence in this case there exists a strictly sincere ballot that yields an outcome which $i$ weakly prefers to $W$.

Case 2. $W$ is strictly preferred to all nonempty subsets of pivotal candidates, that is, $W \succ P^{\prime}$ for all $P^{\prime} \subseteq P$. 
In this case $B$ cannot involve approving of any pivotal candidate. To see this, note that $B \cap P \neq \emptyset$ implies $W \subseteq P$, a contradiction since by assumption $W$ is strictly preferred to all subsets of pivotal candidates. Thus, $B \cap P=\emptyset$ and the set of winners contains all pivotal candidates, i.e. $P \subseteq W$, and possibly some subpivotal candidates. Hence, we can write $W=P \cup S^{\prime}$ for some $S^{\prime} \subseteq S \cap B$ with $S^{\prime}$ non-empty, since $S^{\prime}=\emptyset$ would implies $W=P$, a contradiction since $W \succ P$.

Since $B$ is not strictly sincere, we have $\iota(B) \neq \emptyset$. We distinguish three subcases. Case 2.1. $\max (X \backslash B) \cap(P \cup S)=\emptyset$

In this case, no element of $\max (X \backslash B)$ is pivotal or subpivotal. We can simply add all elements of $\max (X \backslash B)$ to the ballot to obtain a new ballot $B^{*}=B \cup$ $\max (X \backslash B)$. This new ballot is strictly more sincere than $B$ by (S1), while the set of winners remains unchanged, $W^{B^{*}}=W$.

Case 2.2. $\max (X \backslash B) \cap P \neq \emptyset$

In this case, one of the top non-approved alternatives is pivotal. Let $b \in \iota(B)$. The ballot $B^{*}=B \backslash\{b\}$ is strictly more sincere than $B$ by the claim above. The set of winners is $W^{B^{*}}=W \backslash\{b\}$. It remains to show that this set is weakly preferred to $W$ by $i$.

- If $b \notin W$, then the outcome is not affected, that is, $W^{B^{*}}=W$.

- If $b \in W$, then the outcome is $W^{B^{*}}=W \backslash\{b\}$. Let $c \in \max (X \backslash B) \cap P$. By assumption (Case 2) we must have $W \succ\{c\}$. Since $\{c\} \succeq\{b\}$ by (WXT) (which is implied by condition (R) by Lemma 3), it follows that $W \succ\{b\}$ by transitivity. Applying (DEL) we obtain $W \backslash\{b\} \succeq W$ as desired.

Case 2.3. $\max (X \backslash B) \subseteq S$

Finally, we consider the case where all elements of $\max (X \backslash B)$ are subpivotal. First, suppose $\iota(B) \cap S=\emptyset$. In this case, we can just remove all elements in $\iota(B)$ from the ballot (recall that also $B \cap P=\emptyset$ ) and set $B^{*}=B \backslash \iota(B)$, which does not affect the set of winners. Since $\iota\left(B^{*}\right)=\emptyset$, it follows that $B^{*}$ is a strictly sincere ballot.

Second, suppose $\iota(B) \cap S \neq \emptyset$. Let $b \in \max (\iota(B) \cap S)$. By (RP) one of the following three cases holds.

- $W \backslash\{b\} \succeq W$ and $W \neq\{b\}$. Then, the ballot $B^{*}=B \backslash\{b\}$, which is strictly more sincere than $B$ by the claim above, yields the set of winners $W^{B^{*}}=W \backslash\{b\} \succeq W$. 
- $W \cup D \succeq W$ for each $c \in X$ with $c \succeq b$ and $D \subseteq I_{\succeq}(c)$. In this case, let $c \in \max (X \backslash B)$ and note that $\max (X \backslash B) \subseteq I_{\succeq}(c)$ and $c \succeq b$. Then, the ballot $B^{*}=B \cup \max (X \backslash B)$ delivers $W^{B^{*}}=W \cup \max (X \backslash B) \succeq W$ as the set of winners. It remains to show that $B^{*}$ is strictly more sincere than $B$. Let $b^{\prime} \in \max \left(X \backslash B^{*}\right)$ and $b^{*} \in \max (X \backslash B)$. Since $X \backslash B^{*} \subseteq X \backslash B$, it follows that $b^{*} \succeq b^{\prime}$. If $b^{*} \sim b^{\prime}$, then $b^{\prime} \in \max (X \backslash B)$, contradicting $b^{\prime} \in X \backslash B^{*}=X \backslash(B \cup \max (X \backslash B))$. Thus we must have $b^{*} \succ b^{\prime}$. This shows that $B^{*}$ is strictly more sincere than $B$ by $(\mathrm{S} 1)$.

- $(W \backslash\{b\}) \cup D \succeq W$ for each $c \in X$ with $c \succ b$ and $D \subseteq I_{\succeq}(c)$, and also $W \cup D \succeq W$ for each $D \subseteq I_{\succeq}(b)$. First, suppose there is $c \in \max (X \backslash B)$ with $c \succ b$ and note that $\max (X \backslash B) \subseteq I_{\succeq}(c)$. Then setting $B^{*}=(B \backslash\{b\}) \cup$ $\max (X \backslash B)$ delivers the set of winners $W^{B^{*}}=(W \backslash\{b\}) \cup \max (X \backslash B)$, which is weakly preferred to $W$ by hypothesis. To see that $B^{*}$ is strictly more sincere than $B$, let $b^{\prime} \in \max \left(X \backslash B^{*}\right)$ and $b^{*} \in \max (X \backslash B)$. We have $X \backslash B^{*}=(X \backslash(B \cup \max (X \backslash B))) \cup\{b\}$, hence either $b^{\prime}=b$ or $b^{\prime} \in$ $\max \left(X \backslash(B \cup \max (X \backslash B))\right.$. If $b^{\prime}=b$, then since $\max (X \backslash B) \subseteq I_{\succeq}(c)$ and $c \succ b$, it follows that $b^{*} \succ b^{\prime}$. On the other hand, if $b^{\prime} \in \max (X \backslash(B \cup \max (X \backslash B)))$, then since $X \backslash(B \cup \max (X \backslash B)) \subseteq X \backslash B$ and $b^{\prime} \notin \max (X \backslash B)$, it follows that $b^{*} \succ b^{\prime}$ as well. Thus $B^{*}$ is strictly more sincere than $B$ by (S1).

Now, suppose that $c \sim b$ for all $c \in \max (X \backslash B)$. In this case, we must have $\max (X \backslash B) \subseteq I_{\succeq}(b)$ and thus $W \cup \max (X \backslash B) \succeq W$ by hypothesis. Setting $B^{*}=B \cup \max (X \backslash B)$ yields the set of winners $W^{*}=W \cup \max (X \backslash B) \succeq W$. To see that $B^{*}$ is strictly more sincere than $B$, let $b^{\prime} \in \max \left(X \backslash B^{*}\right)$ and $b^{*} \in \max (X \backslash B)$. Since $X \backslash B^{*} \subseteq X \backslash B$ and $\max (X \backslash B) \cap\left(X \backslash B^{*}\right)=\emptyset$, it follows that $b^{*} \succ b^{\prime}$. Hence, $B^{*}$ is strictly more sincere than $B$ by (S1).

Thus, we have proven that, for any given ballot $B$, there is a strictly more sincere ballot $B^{*}$ such that the associated outcome $W^{B^{*}}=f^{A}\left(B^{*}, B_{-i}\right)$ is weakly preferred to $W$ by $i$. In particular, for all nonempty subsets of pivotal candidates $P^{\prime} \subseteq P$ it holds that $W^{B^{*}} \succeq W \succ P$, hence $B^{*}$ and $W^{B^{*}}$ fall into Case 2 as well. This allows us to iterate the argument, and because the set of alternatives $X$ is finite we obtain a strictly sincere ballot that is a weak improvement over $W$ after a finite number of steps.

To complete the proof of (a), choose a best response to $B_{-i}$ under the preference over sets of candidates, which exists since the number of ballots is finite. If that best response is not strictly sincere, by the argument above there exists a weak 
improvement in the form of a strictly sincere ballot, which is necessarily also a best response.

It remains to show part (b). In the proof of (a), note that whenever $B^{*} \neq X$, the ballot $B^{*}$ is a nontrivial, strictly sincere ballot. The proof of (b) will be complete if we can show that it is also possible to improve upon $B$ by switching to a nontrivial, strictly sincere ballot in the extreme case where $B^{*}=X$, which is then strictly sincere but trivial.

Suppose $B^{*}=X$. Clearly $P \neq \emptyset$ and it follows that $P \cap B^{*}=P \neq \emptyset$. Hence, the set of winners $W^{B^{*}}$ is given by $P$. Consider the strictly sincere ballot $\hat{B}=U_{\succeq}(\max (P))$ with associated set of winners $\max (P) \succeq P=W^{B^{*}}$. Note that $\hat{B} \neq B^{*}$ unless $P=X$ and all alternatives in $P$ are indifferent for $i$, which is impossible since $i$ 's preferences over candidates are nontrivial. Hence $\hat{B}$ is a nontrivial, strictly sincere ballot with set of winners $W^{\hat{B}} \succeq W^{B^{*}}$.

Theorem 1 provides a simple formalization of the intuition that Approval Voting favors sincerity. Specifically, independently of what the beliefs of a voter might be, the set of best replies under Approval Voting always contains a (strictly) sincere ballot. This property holds in the general case where voters are endowed with preferences which are not necessarily strict. Of course, this result does not guarantee that sincere ballot-casting is a Nash equilibrium, since voters might have multiple sincere ballots. Still, it shows that, under Approval Voting, voters never have a strict incentive to deviate to insincere ballots.

\section{The case of dichotomous preferences}

Brams and Fishburn (1978) studied preference extensions based on conditions (P) and $(\mathrm{R})$. They considered the extreme case of dichotomous preferences, where each voter has exactly two indifference classes. This is a natural benchmark for Approval Voting because, for each voter, candidates are naturally partitioned into two classes, along the intended lines of approval and disapproval. For this case, condition $(\mathrm{R})$ implies deletion and replacement, and hence the latter two are redundant for Theorem 1.

Lemma 4. If preferences are dichotomous, condition $(R)$ implies deletion and replacement.

Proof. Let $\succeq$ be dichotomous on $X$. Then, there exist $X_{H}, X_{L} \subseteq X$ with $X_{H} \cap$ $X_{L}=\emptyset$ and $X_{H} \cup X_{L}=X$ such that $x \succ y$ for each $x \in X_{H}, y \in X_{L}, x \sim x^{\prime}$ for all $x, x^{\prime} \in X_{H}$, and $y \sim y^{\prime}$ for all $y, y^{\prime} \in X_{L}$. 
Consider deletion first. Let $\emptyset \neq A \in \mathcal{B}$ and $a \in X$ such that $A \succ\{a\}$. It follows that $a \in X_{L}$. To see this, suppose $a \in X_{H}$. Taking $X_{1}=\{a\}, X_{2}=\emptyset$, and $X_{3}=A$ in (R) implies $\{a\} \succeq A$, in contradiction to $A \succ\{a\}$. Hence $a \in X_{L}$. Let now $X_{1}=\emptyset, X_{2}=A \backslash\{a\}$, and $X_{3}=\{a\}$, and note that $X_{2} \neq \emptyset$ because $A \succ\{a\}$. Applying (R) again yields $A \backslash\{a\} \succeq\{a\}$, hence deletion holds.

To see replacement, let $a \in A \in \mathcal{B}$. First, suppose $a \in X_{L}$. If $A \neq\{a\}$, applying (R) to $X_{1}=\emptyset, X_{2}=A \backslash\{a\}$, and $X_{3}=\{a\}$ yields $A \backslash\{a\} \succeq\{A\}$ and (RP.1) holds. If $A=\{a\}$, then for $b \in X$ with $b \succeq a$ and $D \subseteq I_{\succeq}(b)$ applying (R) to $X_{1}=D, X_{2}=A$, and $X_{3}=\emptyset$ yields $A \cup D \succeq A$, showing (RP.2). Next, suppose $a \in X_{H}$. Let $b \in X$ with $b \subseteq a$ and $D \subseteq I_{\subseteq}(b)$. It follows $a \sim b$ and $D \subseteq X_{H}$. Applying (R) to $X_{1}=D, X_{2}=A$, and $X_{3}=\emptyset$ yields $A \cup D \succeq A$, showing (RP.2). Hence, we have either (RP.1) or (RP.2), completing the proof of replacement.

Under dichotomous preferences there are in general many sincere ballots, since a voter can approve of all his "better" candidates and part of the "worse" ones without violating sincerity. In contrast, under dichotomous preferences each voter has exactly one strictly sincere ballot, which coincides with his or her upper indifference class. Brams and Fishburn (1978, Corollary 1) show that this is the only strategy that is not weakly dominated. It follows from Theorem 1 that this sincere ballot is a best response against any constellation of ballots of other voters. By Lemma 4, this holds under just condition (R). We obtain a simple version of Brams and Fishburn (1978) strategy-proofness result.

Corollary 1. If all voters have dichotomous preferences over candidates and preferences over sets fulfilling condition ( $R$ ), casting the (unique) strictly sincere ballots constitutes a Nash Equilibrium in non-weakly dominated strategies.

Brams and Fishburn (1978) established this result under conditions (R) and (P). Our Corollary implies that condition (P) was not necessary.

\section{Relation to other properties and results}

\subsection{Cardinal utilities and random tie-breaking}

Consider voters with preferences that are based on utility functions. Assuming that ties among sets of winners are broken uniformly, the expected utility of a set of alternatives is well-defined. Assuming voters maximize expected utility preferences over sets of alternatives are naturally defined. The next result shows that this classical example of a preference extension to sets satisfies condition (R), deletion, and replacement. 
Proposition 1. Consider a voter who is an expected-utility maximizer expecting uniform tie-breaking. Then the voter's preferences over sets of alternatives satisfy condition ( $R$ ), deletion, and replacement.

Proof. Let $\succeq$ be the set preferences of a voter who is an expected-utility maximizer expecting uniform tie-breaking. Let $u: X \longrightarrow \mathbb{R}_{+}$be the voters utility function. We denote the expected utility of a set $A \subseteq X$ by $u(A)=\sum_{a \in A} \frac{1}{|A|} u(a)$.

Consider monotonicity first. Let $A, B \in \mathcal{B}$ with $B \neq \emptyset$ and $A \subseteq U_{\succeq}(B)$. It follows, $u(a) \geq u(b)$ for all $a \in A, b \in B$ and hence $u(A) \geq u(B)$. Further, $u(A \cup B)=\frac{|A| u(A)+|B| u(B)}{|A|+|B|} \geq \frac{|A| u(B)+|B| u(B)}{|A|+|B|}=u(B)$, that is, $A \cup B \subseteq B$ and (MN1) holds. To see (MN2), let $A \subseteq B$ and $\emptyset \neq B \backslash A \subseteq U_{\succeq}(A)$. It follows $u(B \backslash A) \geq u(A)$ Thus $u(B)=\frac{|B \backslash A| u(B \backslash A)+|A| u(A)}{|B \backslash A|+|A|} \leq \frac{|B \backslash A| u(B \backslash A)+|A| u(B \backslash A)}{|B \backslash A|+|A|}=u(B \backslash A)$, that is, $B \backslash A \succeq B$ and (MN2) holds.

Next, we show deletion. Let $a \in A$ such that $A \succ\{a\}$. This is only possible if $a$ yields below average utility among the alternatives in $A$. It follows that average utility can only increase, when $a$ is removed from $A$, that is, $A \backslash\{a\} \succeq A$. Hence, (DEL) holds.

We now turn to (RP). Let $A \neq \emptyset$ and $a \in A$. If $u(a) \leq u(A)$, then $u(A \backslash$ $\{a\}) \geq u(A)$ and it follows that $A \backslash\{a\} \succeq A$, that is, (RP.1) holds. Now suppose $u(a)>u(A)$ and let $D \subseteq I_{\succeq}(b)$ for some $b \succeq a$ (if no such element exists, then (RP.2) holds trivially). It follows, $u\left(b^{\prime}\right) \geq u(a)>u(A)$ for all $b^{\prime} \in D$. Thus, $D \backslash A \neq \emptyset$ and $u(D \backslash A)>u(A)$. It follows that $u(A \cup D)=\frac{|A| u(A)}{|A|+|D \backslash A|}+\frac{|D \backslash A| u(D \backslash A)}{|A|+|D \backslash A|}>$ $\frac{|A| u(A)}{|A|+|D \backslash A|}+\frac{|D \backslash A| u(A)}{|A|+|D \backslash A|}=u(A)$, which shows $A \cup D \succeq A$. Hence, (RP.2) holds in this case. Taken together, this shows that (RP) holds.

As a consequence, we obtain that under Approval Voting with uniform tiebreaking, upon learning the ballots of the other voters, a voter who is an expectedutility maximizer will always have a best response that is strictly sincere. We have just shown that the (not necessarily strict) preferences of such a voter satisfy condition (R), deletion, and replacement, which allows us to apply Theorem 1. In the following example, we show that the preferences of such a voter do not in general satisfy the stronger condition (SIM).

Remark 1. The preferences over sets of a voter maximizing expected utility assuming that ties are broken at random (uniformly) do not satisfy (SIM). To see this, let $X=\{x, y, z, t\}$ and consider a voter with utility function given by $u(x)=7$, $u(y)=u(z)=6$, and $u(t)=0$. Under (SIM), the set $A=\{x, t\}$ should be at least weakly preferred to $A^{\prime}=\{y, z, t\}$. However, random tie-breaking and expected utility yield $U(A)=\frac{1}{2} 7+\frac{1}{2} 0=3.5<4=\frac{2}{3} 6+\frac{1}{3} 0=U\left(A^{\prime}\right)$. 


\subsection{Relation to the Gärdenfors Principle}

The following pair of properties first appeared in Gärdenfors (1976) and are jointly referred to as the Gärdenfors Principle (GAR). ${ }^{4}$

(GF1) $A \cup\{b\} \succ A$ if $b \succ a$ for all $a \in A$.

(GF2) $A \succ A \cup\{b\}$ if $a \succ b$ for all $a \in A$.

We note that although they capture a similar concept as condition (R), (GAR) and $(\mathrm{R})$ are independent in the sense that none implies the other.

Remark 2. Condition (R) implies neither (GF1) nor (GF2). Let $X=\{a, b\}$ with $a \succ b$. First, consider the preference over sets given by $\{a\} \succ\{a, b\} \sim\{b\}$. It is easy to see that these satisfy (R), but violate (GF1). Second, assume that preferences over sets are given by $\{a\} \sim\{a, b\} \succ\{b\}$. Again $(\mathrm{R})$ is satisfied, but (GF2) does not hold.

Remark 3. (GAR) does not imply condition (R). Let $X=\{a, b, c\}$ with $a \succ b \sim c$. Consider the following preferences over sets: $\{a\} \succ\{a, b\} \sim\{a, c\} \succ\{a, b, c\} \succ$ $\{b\} \sim\{c\} \succ\{b, c\}$. Those satisfy (GAR), but not condition (R). To see this, note that (MN1) would imply that adding $b$ to $\{c\}$ results at least in a weak improvement, that is, $\{b, c\} \succeq\{c\}$, a contradiction.

\subsection{Endriss' conditions and the case of strict preferences}

Endriss (2013) considers the case of strict preferences only, that is, voters are not allowed to be indifferent among any two candidates. In this case, given preferences $\succ$ and any nonempty set of candidates $A$, the best and worst candidates in $A$ according to $\succ$ are always unique and well-defined (because the set of candidates is finite). Slightly abusing notation, we denote them by $\max (A)$ and $\min (A)$, respectively. Endriss (2013) studies a number of properties, some of which are weaker than our conditions, and some of which are stronger.

Consider the following properties.

$(\mathrm{MAX})\{\max (A)\} \succeq A$.

(MIN) $A \succeq\{\min (A)\}$.

(WRP) $(A \backslash\{a\}) \cup\{b\} \succeq A$ or $A \cup\{b\} \succeq A$ or $A \neq\{a\}$ and $A \backslash\{a\} \succeq A$ if $b \succ a$.

\footnotetext{
${ }^{4}$ To economize notation, all properties from this point on are understood to be stated for all ballots and all elements as appropriate.
} 
Property (MAX) states that getting only the best element of a set is at least as good as the whole set. Similarly, (MIN) requires that a set is at least as good as the singleton set containing only its worst element. (WRP) is a weak version of the replacement axiom of Puppe (1995), which characterized a proposal of Sen (1991). Intuitively, it requires for any pair of strictly ranked alternatives that at least one of the following operations weakly improves any given set of alternatives: replacing the worse alternative of the two (given it is in the set) by the better alternative, adding the better alternative to the set, or removing the worse alternative from the set.

The following theorem is the main result of Endriss (2013).

Theorem 2 (Endriss, 2013). Assume the preferences of a voter are strict and satisfy (EXT), (MAX), (MIN), (WRP), and (DEL). Then, given the ballots of other voters, a voter will always have a sincere best response.

As mentioned above, (EXT) is not implied by (SIM) as it states strict preference. However, (SIM) implies all the other properties required for Theorem 2. We first show that already the weaker condition (R) implies (MAX) and (MIN), and as a consequence so does (SIM).

Proposition 2. Assume strict preferences. Then, condition (R) implies (MAX) and $(M I N)$.

Proof. Let $\succeq$ be a preference over sets satisfying condition (R).

$(\mathrm{MAX})$. Let $B \in \mathcal{B}$ and set $A=B \backslash\{\max (B)\} \subseteq B$. It follows, $B \backslash A=$ $\{\max (B)\} \subseteq U_{\succ}(A)$ and $B \backslash A \neq \emptyset$, applying (MN2) to $A$ and $B$ yields $\{\max (B)\}=$ $B \backslash A \succeq B$. Hence, (MAX) holds.

$(\mathrm{MIN})$. Let $B \in \mathcal{B}$ and set $A=B \backslash\{\min (B)\}$. It follows, $A \subseteq U_{\succeq}(\{\min (B)\})$ and $B=\{\min (B)\} \cup A$. Applying $(\mathrm{MN} 1)$ to $\{\max (B)\}$ and $A$ yields $\{\min (B)\} \cup$ $A \succeq\{\min (B)\}$. Hence, $B \succeq\{\min (B)\}$ and (MIN) holds.

In Lemma 2 we have shown that (SIM) implies condition (R), (DEL), and (RP). Clearly, (RP) implies (WRP), that is, the replacement axiom used in our main result is stronger than (WRP). However, for the case of strict preferences $(\mathrm{RP})$ reduces to (WRP). Further, (SIM) also implies (WRP) and we obtain the following result.

Proposition 3. Assume strict preferences. Then, (SIM) implies (MAX), (MIN), (WRP), and (DEL).

However, (WRP), and thus (RP), is not implied by condition (R) as the following remark shows. 
Remark 4. Condition (R) does not imply (WRP). For example, let $X=\{a, b, c, d\}$ with $a \succ b \succ c \succ d$ and consider the following preferences over sets $\succeq$ given by $\{a\} \succ\{a, b\} \succ\{a, b, c\} \succ\{a, c\} \succ\{a, c, d\} \succ\{a, b, d\} \succ\{a, d\} \succ\{a, b, c, d\} \succ$ $\{b\} \succ\{b, c\} \succ\{b, d\} \succ\{b, c, d\} \succ\{c\} \succ\{c, d\} \succ\{d\}$. For these preferences it can be verified that condition (R) holds, but (WRP) is not satisfied for $A=\{a, c, d\}$ and $b \succ c$ since $\{a, c, d\} \succ A \cup\{b\}=X,\{a, c, d\} \succ A \backslash\{c\}=\{a, d\}$, and $\{a, c, d\} \succ A \backslash\{c\} \cup\{b\}=\{a, b, d\}$.

By the last proposition, it follows that if a preference over sets of candidates satisfies (EXT) and (SIM), Theorem 2 applies and sincere best responses exist. For the particular case of strict preferences, sincerity and strict sincerity coincide, and hence, for this case, Theorem 2 would imply Theorem 1 if condition (EXT) were added to the latter's hypotheses. On the other hand, Theorem 1 is stronger than Theorem 2 because, first, it applies to the general case where preferences are not necessarily strict; second, it proves strict sincerity rather than just sincerity even when those concepts differ; and third, it does not require condition (EXT).

Remark 5. The combination of hypotheses in Theorem 2, that is, (EXT), (MAX), (MIN), (WRP), and (DEL), does not imply (SIM). For example, consider the preferences of a voter endowed with a utility function who evaluates sets as if ties are broken uniformly at random. It is clear that (EXT) is satisfied (for details see Endriss, 2013, proof of Theorem 9). By Proposition 1 such preferences satisfy condition (R), deletion and replacement, hence (MAX), (MIN), and (WRP) hold as well. In particular, (EXT), (MAX), (MIN), (WRP), and (DEL) hold as well for the example in Remark 1 where we have already seen that (SIM) does not hold.

However, it can be shown that, in the case of strict preferences, (EXT), (MAX), and (MIN), together with the extreme "optimism" assumption that $A \sim\{\max (A)\}$ (Taylor, 2005), do imply (SIM). An analogous result applies for the "pessimism" assumption that $A \sim\{\min (A)\}$. Although the proof is not immediate, it can also be shown that property (SIM) is also implied (for strict preferences) by the combination of (GAR) and property (MMX) introduced by Kannai and Peleg (1984), i.e. $A \sim\{\max (A), \min (A)\}$.

\section{Conclusion}

We have established a non-manipulability result for Approval Voting which applies to arbitrary preferences (encompassing the particular cases of strict and dichotomous preferences) and provides a formalization for the intuitive idea that voters lack incentives to misrepresent their preferences under Approval Voting. We have 
provided a set of consistency conditions for an extension of a voter's preference over candidates to sets of candidates that, if fulfilled, guarantee that a voter will always have a strictly sincere best response given the ballots of the other voters. Our result is stronger than previous ones because, first, it holds for arbitrary, not necessarily strict preferences over candidates, and, second, we use a more stringent concept of sincerity that requires that every approved candidate is strictly preferred to every disapproved one.

\section{References}

Alós-Ferrer, C. (2006). A Simple Characterization of Approval Voting. Social Choice and Welfare, 27(3):621-625.

Alós-Ferrer, C. and Granić, Đ.-G. (2010). Approval Voting in Germany: Description of a Field Experiment. In Laslier, J.-F. and Sanver, M. R., editors, Handbook on Approval Voting. Springer.

Alós-Ferrer, C. and Granić, Đ.-G. (2012). Two Field Experiments on Approval Voting in Germany. Social Choice and Welfare, 39(1):171-205.

Alós-Ferrer, C. and Granić, Đ. G. (2015). Political Space Representation with Approval Data: the German Experience. Electoral Studies, 39(1):56-71.

Baujard, A. and Igersheim, H. (2010). Framed Field Experiments on Approval Voting: Lessons from the 2002 and 2007 French Presidential Elections. In Laslier, J.-F. and Sanver, M. R., editors, Handbook on Approval Voting. Springer.

Brams, S. J. and Fishburn, P. C. (1978). Approval Voting. The American Political Science Review, 72(3):831-847.

Brams, S. J. and Fishburn, P. C. (2005). Going from Theory to Practice: The Mixed Success of Approval Voting. Social Choice and Welfare, 25(2):457-474.

De Sinopoli, F., Dutta, B., and Laslier, J. F. (2006). Approval Voting: Three Examples. International Journal of Game Theory, 35:27-38.

Endriss, U. (2013). Sincerity and Manipulation under Approval Voting. Theory and Decision, 74(3):335-355.

Fishburn, P. C. (1978a). Axioms for Approval Voting: Direct Proof. Journal of Economic Theory, 19(1):180-185.

Fishburn, P. C. (1978b). Symmetric and Consistent Aggregation with Dichotomous Voting. In Laffont, J.-J., editor, Aggregation and Revelation of Preferences. North-Holland.

Gärdenfors, P. (1976). Manipulation of Social Choice Functions. Journal of Economic Theory, 13(2):217-228. 
Gibbard, A. (1973). Manipulation of Voting Schemes: A General Result. Econometrica, 41(4):587-601.

Kannai, Y. and Peleg, B. (1984). A Note on the Extension of an Order on a Set to the Power Set. Journal of Economic Theory, 32(1):172-175.

Laslier, J.-F. and Van der Straeten, K. (2008). A Live Experiment on Approval Voting. Experimental Economics, 11(1):97-105.

Puppe, C. (1995). Freedom of Choice and Rational Decisions. Social Choice and Welfare, 12(2):137-153.

Satterthwaite, M. A. (1975). Strategy-Proofness and Arrow's Conditions: Existence and Correspondence Theorems for Voting Procedures and Social Welfare Functions. Journal of Economic Theory, 10(2):187-217.

Sen, A. (1991). Welfare, Preference and Freedom. Journal of Econometrics, $50(1): 15-29$.

Taylor, A. D. (2005). Social Choice and the Mathematics of Manipulation. Cambridge University Press.

Wolitzky, A. (2009). Fully Sincere Voting. Games and Economic Behavior, 67:720735 . 